\title{
The Ys and wherefores of protein kinase autoinhibition
}

\section{Authors}

Richard Bayliss*, Tamanna Haq, Sharon Yeoh

Contact details:

Department of Biochemistry, University of Leicester, Lancaster Road, LE1 9HN, United Kingdom.

* To whom correspondence should be addressed: Richard.bayliss@le.ac.uk

\begin{abstract}
Protein phosphorylation is a key reaction in the regulation of cellular events and is catalyzed by over 500 protein kinases in humans. The activities of protein kinases are strictly controlled through a diverse set of mechanisms. Structural studies have shown that the conformation adopted by kinases in their active state are highly similar, whereas inactive kinases can adopt a variety of conformations. Many kinases are maintained in a catalytically inactive state through autoinhibition. This involves a conformation of the kinase active site that is unable to support catalysis and requires activation through a signal such as binding of a regulatory protein. In this review, we briefly summarize some of the well-established autoinhibitory mechanisms and then focus on a relatively unexplored mode of autoinhibition that was first discovered in the Nek family of kinases and is also relevant to IRE1. This involves a tyrosine side-chain that blocks the active site and which must undergo a conformational change to enable kinase activity. We have termed this the Tyr-down autoinhibitory mechanism. We summarise the evidence for this mechanism and describe its role in kinase inhibitor design. Finally, we survey the kinome to identify other kinases with the potential to be governed by an autoinhibitory Tyr-down mechanism.
\end{abstract}

\section{Keywords}

Phosphorylation, structural biology, signaling, regulation

\section{Introduction}

Protein kinases catalyse the transfer of the terminal $\gamma$-phosphate group from ATP to the hydroxyl group of serine, threonine or tyrosine residues on protein substrates. This reaction is at the heart of cellular signaling processes and is frequently altered in diseases such as cancer [1]. The catalytic activity of protein kinases is strictly controlled through a variety of mechanisms, including activation by phosphorylation and/or protein binding partners, autoinhibition and inhibition by protein binding partners [2]. Very commonly, kinase activity is governed by the presence of a phosphorylated residue within a flexible region called the activation loop, activation segment or T-loop [3]. The phosphorylation 
of this residue can be catalyzed by an upstream kinase, as happens in signaling cascades, or can be an auto-catalytic event, as happens in receptor kinases [4, 5].

Kinase auto-phosphorylation implies inhibitory mechanisms that prevent untimely kinase activation. One potential mechanism is autoinhibition, a safety feature built into the kinase itself that prevents it from undergoing autophosphorylation until triggered by an external stimulus. According to the model depicted in Figure 1, a newly-synthesized kinase defaults to this autoinhibited state. An external stimulus releases the kinase from autoinhibition into a preactive state that is capable of auto-phosphorylation but is inefficient at phosphorylation of substrates. Alternatively, an upstream kinase might catalyse the activating phosphorylation event. The phosphorylated kinase then efficiently carries out the phosphorylation of protein substrates.

Kinase autoinhibition operates through a variety of mechanisms. Some kinases possess additional, non-kinase domains that block catalytic activity [6]. For example, the regulatory domain of PKC has a pseudosubstrate sequence that inhibits kinase activity [7]. The juxta-membrane domain of FLT3 forms an extensive interaction with the catalytic domain, locking the kinase in an autoinhibited conformation [8]. Src-family kinases are autoinhibited through interactions between the $\mathrm{SH} 2-\mathrm{SH} 3$ region and the catalytic domain $[9,10]$. Many kinases have autoinhibition built into their catalytic domains, a feature that is the focus of this review.

\section{Structural mechanisms of protein kinase activation}

Before we probe the structural biology of the protein kinase autoinhibition mechanisms, we should briefly review the key features of the kinase catalytic domain. Protein kinase A has served as the archetypal model for the catalytic domain of protein kinases ever since the first crystal structure of this kinase domain was determined, in its active form, phosphorylated on the activation loop [11]. Subsequent structures of protein kinases, such as CDK2, confirmed that there is a conserved protein kinase fold that undergoes conformational changes relating to the regulation of its catalytic activity [12,13]. Protein kinase domains comprise two sub-domains connected by a flexible hinge region (Figure $2 \mathrm{~A}$ ). The $\mathrm{N}$-terminal lobe ( $\mathrm{N}$-lobe) is made up of a 5 -stranded $\beta$-sheet and (usually) two $\alpha$-helices, of which the $\alpha \mathrm{C}$-helix is the structurally most conserved, whereas the larger C-lobe is mainly $\alpha$-helical. There are variations on this core fold, and many kinase domains incorporate extensions that form a structured part of the kinase domain. A deep cleft between the two lobes forms the ATP binding site. The molecular recognition of ATP and protein substrates and the kinase catalytic mechanism of kinases, have been extensively reviewed elsewhere and we will not discuss these details here [14]. Of more immediate relevance to this review are the set of highly conserved motifs and structural elements that form the catalytic core and key regulatory features of protein kinases (Figure 2). Protein crystallography has revealed the structural differences between protein kinases in active and inactive states within these conserved motifs [2, 6, 15-17]. 


\section{Catalytic residues: $H R D$ and $D F G$}

The His-Arg-Asp (HRD) and Asp-Phe-Gly (DFG) motifs are highly conserved tripeptide motifs that are located in the active site and the Asp residues in these motifs contribute to catalysis: the HRD motif Asp side chain acts as a catalytic base that deprotonates the side chain of the substrate residue undergoing phosphorylation; the DFG motif Asp side chain coordinates a magnesium ion that activates the terminal, $\gamma$-phosphate of ATP. These residues must be positioned correctly in order for the kinase to catalyse phosphate transfer. In active kinases the DFG motif adopts the DFG-in conformation, and contacts the HRD motif through a hydrophobic interaction. Many kinases undergo conformational changes that affect the positions of the DFG and HRD motifs. Perhaps the best characterised is the DFG-flip, in which the Phe and Asp side-chains of this motif swap positions. This results in a conformation called DFG-out, in which the kinase is catalytically inactive because the Asp catalytic base is removed from the active site and the Phe side-chain occludes ATP binding [18]. This conformation acts as an autoinhibitory mechanism in the insulin receptor kinase, IRK [19]. Variations on this theme include the DFG-up conformation, observed in Aurora$A$, in which the Asp side-chain moves out of the active site, but the Phe side-chain does not take its place [20].

\section{Lys-Glu salt bridge}

A conserved salt bridge in the N-lobe between a Lys residue on the $\beta 3$-strand and a Glu residue on the $\alpha \mathrm{C}$-helix connects these two secondary structure elements. The Lys side-chain positions the terminal phosphate groups of ATP in the optimal position for catalysis. Another strategy for kinase inactivation involves an outward motion of the $\alpha \mathrm{C}$-helix that breaks this salt-bridge. First noted in the SRC and CDK families of kinases, this mechanism has now been observed more widely and it has been referred to as the CDK/SRC-like inactive conformation (Figure 2B) [16].

\section{Activation loop}

The activation loop spans a sequence starting at the DFG motif and ending at an Ala-Pro-Glu (APE) motif. The activation loop is variable in length and sequence, and is the primary site of regulatory phosphorylation. Unphosphorylated activation loops are typically either disordered, or adopt a conformation that is incompatible with activity. For example, in a CDK/SRC-like inactive conformation the region of the activation loop immediately following the DFGmotif forms an $\alpha$-helix that stabilises the outward position of the $\alpha \mathrm{C}$-helix. A similar autoinhibitory mechanism is at work in EGFR [21].

In a high proportion of kinases, phosphorylation of the activation loop at one or more positions leads to the adoption of a stable conformation compatible with binding of protein substrate. The phosphorylated residue on the activation loop forms interactions with the side chains of basic residues at on at least one of three different positions: i) one amino acid $\mathrm{N}$-terminal to the glutamic acid on the $\alpha \mathrm{C}$-helix; ii) three amino acids C-terminal to the DFG motif; iii) the middle amino acid of the HRD motif (Figure 2C).

\section{Regulatory spine}


A recently defined feature of active kinase structures is a number of hydrophobic residues that form two continuous hydrophobic 'spines' $[17,22]$. One of these spines, called the regulatory or R-spine, is made up of the sidechains of four residues, one from the $\beta 4$-strand in the $\mathrm{N}$-terminal lobe, one from the adjacent $\alpha \mathrm{C}$-helix, one from the middle residue from the DFG motif and one from the first residue from the HRD motif. The relative positions of these four residues are consistent in active kinase states, but divergent in inactive states [23]. For example, in the DFG-out inactive conformation, the R-spine is broken by removal of the Phe side-chain of the DFG-motif, the third residue from the top of the Rspine. In the CDK/SRC-like inactive conformation, the R-spine is broken because the hydrophobic residue on the $\alpha \mathrm{C}$-helix is moved away from the active site (Figure 2D). A systematic evaluation of the biochemical properties of the R-spine residues and number of surrounding, "shell" hydrophobic residues confirmed that the hydrophobic nature of their side chains is essential for catalytic activity and provided other insights of value for understanding kinase regulation and of interest in the development of rational approaches to kinase drug discovery [23]. Interestingly, while the bottom two R-spine residues usually have aromatic side chains, the $\alpha \mathrm{C}$-helix R-spine residue side chain is usually Leu and mutation to bulkier Ile, Met or Phe is sufficient to drive kinase activity [24].

Displacement of the DFG motif, $\alpha \mathrm{C}$-helix and R-spine into positions incompatible with catalysis is part of the regulatory mechanism of many kinases and can also be induced by small molecule inhibitors of kinases. $\alpha \mathrm{C}$-helix out and DFG-out autoinhibited conformations have been extensively reviewed elsewhere [2]. In this review, we focus on a less extensively characterized autoinhibitory mechanism first identified in the crystal structure of Nek7 kinase.

\section{An autoinhibitory Tyr mechanism in Nek7}

Nek7 is a member of the NIMA-related family of kinases (Neks) that number 11 in humans [25]. Together with Nek6 and Nek9, Nek7 functions in the regulation of mitotic spindle assembly during early mitosis [26-32]. Nek7 is activated by Nek9 through two mechanisms: Nek9 phosphorylates Nek7 on Ser195 in the activation loop [26]; the non-catalytic C-terminal domain of Nek9 (Nek9-CTD) binds Nek7 and increases its activity [33]. Nek7 is also able to autophosphorylate through an intermolecular mechanism, which can be accelerated by Nek9-CTD [34].

Nek7 comprises only a kinase catalytic domain with a short N-terminal extension (NTE) of $\sim 30$ aa and, similar to Nek6 but unlike the other human Neks, lacks any additional domains. Full-length human Nek7 in an unphosphorylated, inactive form has been crystallized and its structure has been solved [33]. Nek7 has a classical kinase domain fold, in which the two lobes form a deep pocket that forms the ATP binding site (Figure 3A). The first 20 aa of the NTE was disordered, but the final 10 aa, which is crucial for catalytic activity, was ordered in the crystal structure and caps the N-lobe. Perhaps the most striking feature of the Nek7 structure is the position of the four residues that form the hydrophobic regulatory spine (R-spine). The four positions are Tyr97 ( $\beta 4$-strand), Leu86 ( $\alpha \mathrm{C}$ helix), Leu180 (DLG-motif) and His159 (HRD-motif). Interestingly, the canonical 
"DFG" motif is present as the less common "DLG" in Nek7 kinase. In active kinases, these residues adopt well-defined and conserved positions that support the framework of catalytic residues in the kinase active site [22]. In Nek7, the Rspine is malformed because Tyr97 at the $\beta 4$-strand position is out of the expected position (Figure 3A,B).

The Nek7 structure has several features that are different from what would be expected of an active kinase (Figure 3B). To aid comparison, the structure of an active kinase is shown in Figure 3C and key features are colour-coded. The structure of TA02 kinase was chosen for this comparison because it has a tyrosine (Tyr91) in the R-spine position on the $\beta 4$-strand analogous to Tyr97 of Nek7 [35].

Active kinases form a salt bridge between the side-chains of a Lys on the $\beta 3$ strand and a Glu on the $\alpha \mathrm{C}$-helix, shown on TAO2 as a black dashed line between red side-chains (Figure 3C). This salt-bridge is not present in the Nek7 structure because the $\alpha \mathrm{C}$-helix is rotated out of position and, consequently, Glu82 is over 10 Å distant from Lys63.

An Arg side-chain in the HRD-motif of TAO2 coordinates a phosphorylated serine on the activation loop, shown as a black dashed line between blue side-chains. The equivalent interaction in the Nek7 structure is not formed because Ser195, the site of Nek7phosphorylation, is not phosphorylated. Instead, Arg160 forms a salt-bridge with Glu82. Indeed, the activation loop is disordered between residues 181-198 and in the original structure no density was present for this region.

The R-spine of TAO2 is formed from a linear stack of sidechains: Tyr91-Leu80Phe170-His149, from top to bottom. In contrast, the top three R-spine sidechains of Nek7 are non-linear because the side-chain of Tyr97 is pointing down into the active site and partially displaces the Leu86 side-chain. Displacement of Leu86 is accommodated by rotation and translation of the $\alpha \mathrm{C}$-helix.

In summary, the structure of native Nek7 possesses several features rendering it an inactive kinase. As already mentioned, the salt bridge formed between the Lys on the $\beta 3$-strand and the Glu on the $\alpha \mathrm{C}$-helix is not present. The activation loop is disordered and Ser195 remains unphosphorylated. Tyr97 appears to be locked in an inactive conformation due to its H-bond with Leu180, and also the aromatic ring of Tyr is wedged between the gatekeeper Leu111 and the R-spine residue Leu86, further holding it in this active state. Thus, the structure of inactive Nek7 suggests that Tyr97 has an autoinhibitory function. We predict that an active Nek7 structure would possess the salt bridge between Lys63 and Glu82 as the $\alpha \mathrm{C}$-helix would be rotated in the correct orientation and the resulting active kinase would be phosphorylated at Ser195 and have an ordered activation loop and.

Mutation of Nek7 Tyr97 to Ala, Leu or Phe results in a more active kinase, consistent with an autoinhibitory role of Tyr97[33]. These experiments were carried out using initially unphosphorylated Nek7 proteins, and kinase activity 
was quantified using a generic substrate. It remains to be discovered exactly how these mutations increase kinase activity. The mutations may enhance the overall flexibility of the kinase and thereby destabilize the autoinhibited state, but is this sufficient to generate an active kinase or is a further activation step required? Do they accelerate the rate of kinase auto-phosphorylation or do they increase the activity of the active kinase?

The physiological relevance of the autoinhibitory tyrosine mechanism has not yet been fully explored, but there is evidence that it contributes to cell-cycle regulation of Nek7. Nek7, and the highly related Nek6, which is also downstream of Nek9, have lower levels of kinase activity during mitosis than during interphase. In contrast, mutation of the autoinhibitory tyrosine in Nek6 and Nek7 results in higher levels of activity during both interphase and mitosis [33]. This suggests that autoinhibition is necessary for maintaining low levels of Nek6/7 kinase activity. But how might autoinhibition be released in mitosis? Nek9-CTD increases the activity of wild-type Nek7 to that of the Y97A mutant alone, suggesting this autoinhibitory mechanism is relieved through binding of Nek9-CTD.

It is assumed that phosphorylation of Ser195 on the activation loop of Nek7 is required for full kinase activity. However, the contribution of Ser195 phosphorylation to Nek7 has not been extensively characterized and the kinetic parameters of Nek7 in phosphorylated or unphosphorylated states are unknown. Is the role of Ser195 phosphorylation to drive formation of the active kinase, or does it prevent reversal back to an inhibited state? Could a complex of Nek7 bound to Nek9-CTD achieve a substantial catalytic activity in the absence of Ser195 phosphorylation, as observed in other kinases? [36] Is phosphorylation of Ser195 sufficient to overcome tyrosine autoinhibition or is a coordinated effect of Nek9-CTD and Ser195 phosphorylation required? Which is more important for Ser195 phosphorylation in vivo, autophosphorylation or trans-phosphorylation by Nek9? We are currently addressing these questions in the activation pathway of Nek7. However, two other questions relating to the tyrosine autoinhibition are perhaps of wider interest: does this mechanism contribute to the regulation of other kinases and can it be exploited in the development of new kinase inhibitors?

\section{Targeting autoinhibitory mechanisms in Nek2}

The most obvious place to look for the tyrosine autoinhibition mechanism is in the other members of the Nek family. Of the 11 members in humans, 8 have an equivalent tyrosine (Nek1, Nek2, Nek4, Nek6, Nek8, Nek9, Nek10), whereas the other 3 human Neks (Nek3, Nek5, Nek11) have a phenylalanine in this position. The PDB currently has 24 structures of human Neks - 20 Nek2 structures, 2 Nek7 structures and 2 Nek1 structures. Of these structures, both Nek7 structures and 10 of the Nek2 structures display characteristics of the tyrosine autoinhibitory mechanism.

Nek2 localises to the centrosomes and contributes to the regulation of spindle pole separation by phosphorylation of centrosomal Nek2-associated protein 1 
(C-Nap1) and rootletin [25]. Nek2 phosphorylates C-Nap1 at multiple sites, leading to loss of oligomerization and centrosome association [37]. During G2, Nek2 forms a complex with protein phosphatase 1 (PP1) and mammalian STE20like protein kinase 2 (MST2), and is kept inactive by dephosphorylation [38, 39]. Nek2 is inactivated through autophosphorylation after PP1 is released from the Nek2-MST2 complex [40]. Nek2 has been identified as a potential cancer drug target in cholangiocarcinoma and breast cancer [41-43].

The structure of the Nek2 catalytic domain has been captured in apo-form and bound to a number of different ligands [33, 44-48]. The first structure of human Nek2 was obtained bound to a pyrrole-indolino inhibitor (Figure 4A)[45]. In this structure, Tyr70, which is the equivalent to Tyr97 of Nek7, is clearly in the up position. Reminiscent of the CDK/SRC-like inactive conformation, the $\alpha C$-helix is positioned outwards and the activation loop forms an $\alpha$-helix ( $\alpha \mathrm{T}$ ) that stabilizes the outward position of the $\alpha \mathrm{C}$-helix. Nek2 is found in a DFG-out conformation in the presence of two other series of inhibitors, the hybrid series of potent Nek2 inhibitors and the benzimidazoles upon which the hybrid compounds were based (Figure 4B) $[44,46]$.

With the exception of the activation loop and the residues that interact with the inhibitors, these two inhibitor-bound conformations of Nek2 are similar and the $\mathrm{R}$-spine is disrupted due to displacement of three out of four side chains (Leu59, Phe160 and His139). Structures of apo-Nek2, and Nek2 in complex with ADP or the non-hydrolyzable ATP analogue ATP $\gamma \mathrm{S}$ also show divergent conformations of the DFG-motif [47]. We draw two main conclusions from these structures. First, the activation loop of Nek2 has a remarkable degree of conformational plasticity and, second, the specific conformation adopted can be stabilized inhibitors.

Tyr70 is also clearly in the up position in the ADP-bound structure (Figure 4C). The conformation of Tyr70 is altered when Nek2 is bound to inhibitors based on aminopyrazine or aminopyridine scaffolds (Figure 4D) $[33,48]$. In 7 of these structures, Tyr70 is observed in a down conformation (PDB IDs: 2WQ0, 2XK3, 2XK6, 2XK8, 2XKD, 2XKE, 2XKF), and in 3 structures there is evidence of both Tyr-up and Tyr-down conformations (PDB IDs: 2XK4, 2XK7, 2XKC). However, two further structures of Nek2 bound to aminopyridine compounds show Tyr70 in the up position (PDB IDs: 4A4X, 4AFE) [44].

The fact that the Tyr-down conformation is not observed with apo-Nek2 or Nek2 bound to the pyrrole-indolino inhibitors suggests an induced fit of the binding site by the aminopyrazine/aminopyridine inhibitors. Indeed, the induced fit is thought to occur during the soaking process used to generate the Nek2-inhibitor complex structures: crystals of Nek2-ADP, in which the kinase has a Tyr-up conformation, are soaked with inhibitors over a period of several hours resulting in Nek2-aminopyrazine/aminopyrazine structures in which the kinase has a Tyrdown conformation. The movement is induced through interactions between the inhibitor and the protein, in particular through contacts with the side-chains of Met86 and Tyr70. Upon compound binding, Met86 and Leu59 adopt different positions than in the structure of Nek2 bound to ADP and form a pocket that is 
occupied by Tyr70 in the Tyr-down conformation (Figure 4D). This is very similar to what is observed in Nek7 structures (Figure 3B).

Although the inhibitors can induce a Tyr-down conformation in Nek2, it is not clear that this is important for their potency. Indeed, the most potent Nek2 inhibitors do not induce Tyr-down, but induce a DFG-out conformation and, if we are to develop inhibitors of Nek2 it is not clear which of these conformations, if any, should be targeted. Further studies are needed to resolve which of the autoinhibitory mechanisms suggested by the crystal structures of Nek2 are physiologically relevant: DFG-out, $\alpha C$-helix out or Tyr-down.

\section{Tyr-down autoinhibitory mechanism in IRE1}

IRE1 is resident in the endoplasmic reticulum (ER) and comprises a lumenal domain, a transmembrane region and a cytoplasmic domain that comprises both protein kinase and RNase activities [49]. In response to elevated levels of unfolded protein in the ER, the RNase activity of IRE1 is switched on and results in mRNA splicing of X-box binding protein 1 (XBP1), a transcription factor that promotes the expression of genes encoding ER-resident chaperones. Unfolded protein causes oligomerization of the IRE1 lumenal domain, which drives selfassociation of the cytosolic kinase-RNase domain. This results in autophosphorylation, which is required for RNase activity. Crystal structures of the cytosolic region of human, murine and yeast IRE1 have captured several conformations. Unphosphorylated human IRE1 kinase-RNase domain, crystallized in complex with ADP, revealed an inactive conformation of the kinase, characterized by disruption of the R-spine, loss of the Lys-Glu salt bridge and disordered activation loop (Figure 5A) [50, 51]. Tyr628, the $\beta 4$-strand component of the R-spine equivalent to Nek7 Tyr97, is in the down position and forms a H-bond with the activation loop at Asp711 of the DFG-motif. In contrast, the human kinase-RNase domain crystallized in apo-form exhibited a pre-active kinase conformation, in which the R-spine and Lys-Glu salt bridges are assembled, but the unphosphorylated activation loop is disordered (Figure 5B)[52]. Mutation of Tyr628 to phenylalanine increases the autophosphorylation rate, suggesting that the Tyr-down conformation captured in the structure of ADP-bound human IRE1 is a genuine autoinhibitory state [52]. The fully active form of human IRE1 has not been crystallized, but this state has been captured for the $S$. cerevisiae protein (Figure $5 A)[53,54]$. A full description of the mechanisms of IRE1 RNase regulation and how this is coupled to kinase activity is beyond the scope of this review, and has been extensively covered elsewhere [55].

\section{Prospects for Tyr-down autoinhibitory mechanisms in other human protein kinases}

The presence of a Tyr residue at the top position of the R-spine on the $\beta 4$-strand is a common feature of Neks, found in 8 out of 11 human Neks and the archetypal NimA protein from Aspergillus [33]. We used a bioinformatics approach to ask whether this pattern is reproduced across the human kinome. We found that 56 human kinase domains have a Tyr residues at the equivalent position to Tyr97 of 
Nek7, based on the sequence alignment of human kinase domains [56]. These kinases are not distributed evenly among the nine groups, classified according to sequence comparison of catalytic domains (Table 1, Figure 6A). 29 of these kinases were found in the STE group, 21 in the Other group, 4 in the TK group and 1 each in the RGC and CK groups, and none were found in the remaining groups (AGC, CAMK, CMGC and TKL). Among the Other group of kinases, the presence of a Tyr residue in the $\beta 4$-strand $\mathrm{R}$-spine position is most prevalent in the NEK family, and it is present in more than one member of each of the PEK, IRE, WEE and NAK families.

We examined available structures of STE group kinases to find any examples of a Tyr-down conformation. One of the available structures of oxidative stressresponse kinase 0SR1 exhbits a Tyr-down conformation in one of the four molecules in the asymmetric unit of the crystal (PDB ID: 2VWI, chain A) [57]. As with Nek2 and Nek7, the Tyr78 side-chain points into the active site and packs against the gatekeeper side-chain, Met92. Hydrogen bonds are formed between the hydroxyl of Tyr78 and the DFG-motif at Asp164 and Gly166 (Figure 6B). Strikingly, the region corresponding to the $\alpha \mathrm{C}$-helix is disordered in this chain. In this conformation, the kinase would clearly require a major conformational change in order to gain catalytic activity. The other three chains of OSR1 in the asymmetric unit of the crystals have a Tyr-up conformation, and the $\alpha \mathrm{C}$-helix is ordered (Figure 6C). In these three chains, the activation loop folds into an $\alpha$ helix, closely resembling a CDK/SRC-like autoinhibitory conformation. The Rspine side-chain on the $\alpha \mathrm{C}$-helix, Met67, together with the gatekeeper, Met92, block the Tyr-down pocket. It is unclear why two different conformations of OSR1 have been captured in these structures, but this is a relatively common occurrence in protein crystallography. It would be interesting to probe whether OSR1 has CDK/SRC-like and/or Tyr-down modes of autoinhibition. OSR1 would therefore be a reasonable starting point for a wider investigation into the relevance of Tyr-down autoinhibition in STE group kinases.

A further example is found in the PEK family kinase GCN2, in which the structure of the apo-form shows a Tyr-down conformation in all four chains in the asymmetric unit of the crystal lattice (PDB ID: 1ZYC) [58]. Tyr658, the $\beta 4$-strand component of the R-spine equivalent to Nek7 Tyr97, forms a H-bond with the main chain at the DFG-motif and is sandwiched between Met788, the gatekeeper residue, and Leu647, the $\alpha \mathrm{C}$-helix component of the R-spine (Figure 6D). The Lys-Glu salt bridge is broken, and Glu643 instead forms a salt bridge with Arg834 of the HRD-motif, similar what is observed in Nek7. Indeed, the Tyrdown conformation of GCN2 closely resembles that of Nek7 and most of the key residues are conserved, with the exception of the DFG-motif which has the canonical Phe at position 854 in GCN2, whereas Nek7 has the non-canonical Leu180. Although the structure of GCN2 suggests that it might be regulated by a Tyr-down autoinhibitory mechanism, further studies are required to clarify whether this is physiologically relevant for GCN2 or other PEK family kinases.

\section{Concluding remarks}


Kinase autoinhibition acts to prevent untimely kinase activation and has been exploited in drug discovery. Structural studies using protein crystallography have revealed mechanisms such as DFG out, $\alpha \mathrm{C}$-helix out and Tyr-down that have been demonstrated to contribute to kinase regulation using biochemical methods and cell-based assays. Kinase active and inactive conformations are not static, but exist in equilibrium. Our view of these states is somewhat restricted by the major limitation of protein crystallography: namely, the requirement to trap a defined state in the crystal lattice while, in solution, it is likely that many further conformations are sampled by the kinase.

We do not think it is straightforward to predict whether a given kinase is subject to any particular autoinhibitory mechanism. Indeed, related kinases do not necessarily have the same autoinhibitory mechanism. For example, structural studies on the insulin receptor family of kinases have revealed that while kinases such as TrkA and IRK rely on a DFG-out conformation, kinases such as ALK and Met use a CDK/Src-like mode of autoinhibition [59]. A combination of structural, biochemical and cell biological studies will be required to further delineate the modes of kinase autoinhibition across the kinome.

\section{Acknowledgements}

Research in the Bayliss group relating to this review is funded by grants from Cancer Research UK (C24461/A12772 and C24461/A13231) and the Medical Research Council (MR/L017032/1).

1 Brognard, J. and Hunter, T. (2011) Protein kinase signaling networks in cancer. Curr Opin Genet Dev. 21, 4-11

2 Endicott, J. A., Noble, M. E. and Johnson, L. N. (2012) The structural basis for control of eukaryotic protein kinases. Annu Rev Biochem. 81, 587-613

3 Johnson, L. N. and Lewis, R. J. (2001) Structural basis for control by phosphorylation. Chem Rev. 101, 2209-2242

4 Hubbard, S. R. (2004) Juxtamembrane autoinhibition in receptor tyrosine kinases. Nat Rev Mol Cell Biol. 5, 464-471

5 Dhillon, A. S., Hagan, S., Rath, O. and Kolch, W. (2007) MAP kinase signalling pathways in cancer. Oncogene. 26, 3279-3290

6 Huse, M. and Kuriyan, J. (2002) The conformational plasticity of protein kinases. Cell. 109, 275-282

$7 \quad$ House, C. and Kemp, B. E. (1987) Protein kinase C contains a pseudosubstrate prototope in its regulatory domain. Science. 238, 1726-1728 8 Griffith, J., Black, J., Faerman, C., Swenson, L., Wynn, M., Lu, F., Lippke, J. and Saxena, K. (2004) The structural basis for autoinhibition of FLT3 by the juxtamembrane domain. Mol Cell. 13, 169-178

9 Sicheri, F., Moarefi, I. and Kuriyan, J. (1997) Crystal structure of the Src family tyrosine kinase Hck. Nature. 385, 602-609

$10 \mathrm{Xu}$, W., Doshi, A., Lei, M., Eck, M. J. and Harrison, S. C. (1999) Crystal structures of c-Src reveal features of its autoinhibitory mechanism. Mol Cell. 3, 629-638 
11 Knighton, D. R., Zheng, J. H., Ten Eyck, L. F., Ashford, V. A., Xuong, N. H., Taylor, S. S. and Sowadski, J. M. (1991) Crystal structure of the catalytic subunit of cyclic adenosine monophosphate-dependent protein kinase. Science. 253, 407-414

12 De Bondt, H. L., Rosenblatt, J., Jancarik, J., Jones, H. D., Morgan, D. O. and Kim, S. H. (1993) Crystal structure of cyclin-dependent kinase 2. Nature. 363, 595-602

13 Jeffrey, P. D., Russo, A. A., Polyak, K., Gibbs, E., Hurwitz, J., Massague, J. and Pavletich, N. P. (1995) Mechanism of CDK activation revealed by the structure of a cyclinA-CDK2 complex. Nature. 376, 313-320

14 Johnson, L. N., Noble, M. E. and Owen, D. J. (1996) Active and inactive protein kinases: structural basis for regulation. Cell. 85, 149-158

15 Bayliss, R., Fry, A., Haq, T. and Yeoh, S. (2012) On the molecular mechanisms of mitotic kinase activation. Open Biol. 2, 120136

16 Jura, N., Zhang, X., Endres, N. F., Seeliger, M. A., Schindler, T. and Kuriyan, J. (2011) Catalytic control in the EGF receptor and its connection to general kinase regulatory mechanisms. Mol Cell. 42, 9-22

17 Kornev, A. P. and Taylor, S. S. (2010) Defining the conserved internal architecture of a protein kinase. Biochim Biophys Acta. 1804, 440-444

18 Schindler, T., Bornmann, W., Pellicena, P., Miller, W. T., Clarkson, B. and Kuriyan, J. (2000) Structural mechanism for STI-571 inhibition of abelson tyrosine kinase. Science. 289, 1938-1942

19 Hubbard, S. R., Wei, L., Ellis, L. and Hendrickson, W. A. (1994) Crystal structure of the tyrosine kinase domain of the human insulin receptor. Nature. 372, 746-754

20 Dodson, C. A., Kosmopoulou, M., Richards, M. W., Atrash, B., Bavetsias, V., Blagg, J. and Bayliss, R. (2010) Crystal structure of an Aurora-A mutant that mimics Aurora-B bound to MLN8054: insights into selectivity and drug design. Biochem J. 427, 19-28

21 Zhang, X., Gureasko, J., Shen, K., Cole, P. A. and Kuriyan, J. (2006) An allosteric mechanism for activation of the kinase domain of epidermal growth factor receptor. Cell. 125, 1137-1149

22 Kornev, A. P., Haste, N. M., Taylor, S. S. and Eyck, L. F. (2006) Surface comparison of active and inactive protein kinases identifies a conserved activation mechanism. Proc Natl Acad Sci U S A. 103, 17783-17788

23 Meharena, H. S., Chang, P., Keshwani, M. M., Oruganty, K., Nene, A. K., Kannan, N., Taylor, S. S. and Kornev, A. P. (2013) Deciphering the structural basis of eukaryotic protein kinase regulation. PLoS Biol. 11, e1001680

24 Hu, J., Ahuja, L. G., Meharena, H. S., Kannan, N., Kornev, A. P., Taylor, S. S. and Shaw, A. S. (2015) Kinase regulation by hydrophobic spine assembly in cancer. Mol Cell Biol. 35, 264-276

25 Fry, A. M., O'Regan, L., Sabir, S. R. and Bayliss, R. (2012) Cell cycle regulation by the NEK family of protein kinases. J Cell Sci. 125, 4423-4433 26 Belham, C., Roig, J., Caldwell, J. A., Aoyama, Y., Kemp, B. E., Comb, M. and Avruch, J. (2003) A mitotic cascade of NIMA family kinases. Nercc1/Nek9 activates the Nek6 and Nek7 kinases. J Biol Chem. 278, 34897-34909

27 O'Regan, L. and Fry, A. M. (2009) The Nek6 and Nek7 protein kinases are required for robust mitotic spindle formation and cytokinesis. Mol Cell Biol. 29, 3975-3990 
28 Rapley, J., Nicolas, M., Groen, A., Regue, L., Bertran, M. T., Caelles, C., Avruch, J. and Roig, J. (2008) The NIMA-family kinase Nek6 phosphorylates the kinesin Eg5 at a novel site necessary for mitotic spindle formation. J Cell Sci. 121, 3912-3921

29 Sdelci, S., Bertran, M. T. and Roig, J. (2011) Nek9, Nek6, Nek7 and the separation of centrosomes. Cell Cycle. 10, 3816-3817

30 Sdelci, S., Schutz, M., Pinyol, R., Bertran, M. T., Regue, L., Caelles, C., Vernos, I. and Roig, J. (2012) Nek9 Phosphorylation of NEDD1/GCP-WD Contributes to Plk1 Control of gamma-Tubulin Recruitment to the Mitotic Centrosome. Curr Biol. 22, 1516-1523

31 Yin, M. J., Shao, L., Voehringer, D., Smeal, T. and Jallal, B. (2003) The serine/threonine kinase Nek6 is required for cell cycle progression through mitosis. J Biol Chem. 278, 52454-52460

32 Yissachar, N., Salem, H., Tennenbaum, T. and Motro, B. (2006) Nek7 kinase is enriched at the centrosome, and is required for proper spindle assembly and mitotic progression. FEBS Lett. 580, 6489-6495

33 Richards, M. W., O'Regan, L., Mas-Droux, C., Blot, J. M., Cheung, J., Hoelder, S., Fry, A. M. and Bayliss, R. (2009) An autoinhibitory tyrosine motif in the cellcycle-regulated Nek7 kinase is released through binding of Nek9. Mol Cell. 36, 560-570

34 Dodson, C. A., Yeoh, S., Haq, T. and Bayliss, R. (2013) A kinetic test characterizes kinase intramolecular and intermolecular autophosphorylation mechanisms. Sci Signal. 6, ra54

35 Zhou, T., Raman, M., Gao, Y., Earnest, S., Chen, Z., Machius, M., Cobb, M. H. and Goldsmith, E. J. (2004) Crystal structure of the TAO2 kinase domain: activation and specificity of a Ste20p MAP3K. Structure. 12, 1891-1900 36 Dodson, C. A. and Bayliss, R. (2012) Activation of Aurora-A kinase by protein partner binding and phosphorylation are independent and synergistic. J Biol Chem. 287, 1150-1157

37 Hardy, T., Lee, M., Hames, R. S., Prosser, S. L., Cheary, D. M., Samant, M. D., Schultz, F., Baxter, J. E., Rhee, K. and Fry, A. M. (2014) Multisite phosphorylation of C-Nap1 releases it from Cep135 to trigger centrosome disjunction. J Cell Sci. 127, 2493-2506

38 Helps, N. R., Luo, X., Barker, H. M. and Cohen, P. T. (2000) NIMA-related kinase 2 (Nek2), a cell-cycle-regulated protein kinase localized to centrosomes, is complexed to protein phosphatase 1. Biochem J. 349, 509-518

39 Mardin, B. R., Lange, C., Baxter, J. E., Hardy, T., Scholz, S. R., Fry, A. M. and Schiebel, E. (2010) Components of the Hippo pathway cooperate with Nek2 kinase to regulate centrosome disjunction. Nat Cell Biol. 12,1166-1176

40 Mardin, B. R., Agircan, F. G., Lange, C. and Schiebel, E. (2011) Plk1 controls the Nek2A-PP1gamma antagonism in centrosome disjunction. Curr Biol. 21, 1145-1151

41 Hayward, D. G., Clarke, R. B., Faragher, A. J., Pillai, M. R., Hagan, I. M. and Fry, A. M. (2004) The centrosomal kinase Nek2 displays elevated levels of protein expression in human breast cancer. Cancer Res. 64, 7370-7376 42 Kokuryo, T., Senga, T., Yokoyama, Y., Nagino, M., Nimura, Y. and Hamaguchi, M. (2007) Nek2 as an effective target for inhibition of tumorigenic growth and peritoneal dissemination of cholangiocarcinoma. Cancer Res. 67, 9637-9642 
43 Tsunoda, N., Kokuryo, T., Oda, K., Senga, T., Yokoyama, Y., Nagino, M., Nimura, Y. and Hamaguchi, M. (2009) Nek2 as a novel molecular target for the treatment of breast carcinoma. Cancer Sci. 100, 111-116

44 Innocenti, P., Cheung, K. M., Solanki, S., Mas-Droux, C., Rowan, F., Yeoh, S., Boxall, K., Westlake, M., Pickard, L., Hardy, T., Baxter, J. E., Aherne, G. W., Bayliss, R., Fry, A. M. and Hoelder, S. (2012) Design of potent and selective hybrid inhibitors of the mitotic kinase Nek2: structure-activity relationship, structural biology, and cellular activity. J Med Chem. 55, 3228-3241

45 Rellos, P., Ivins, F. J., Baxter, J. E., Pike, A., Nott, T. J., Parkinson, D. M., Das, S., Howell, S., Fedorov, O., Shen, Q. Y., Fry, A. M., Knapp, S. and Smerdon, S. J. (2007) Structure and regulation of the human Nek2 centrosomal kinase. J Biol Chem. 282, 6833-6842

46 Solanki, S., Innocenti, P., Mas-Droux, C., Boxall, K., Barillari, C., van Montfort, R. L., Aherne, G. W., Bayliss, R. and Hoelder, S. (2011) Benzimidazole inhibitors induce a DFG-out conformation of never in mitosis gene A-related kinase 2 (Nek2) without binding to the back pocket and reveal a nonlinear structure-activity relationship. J Med Chem. 54, 1626-1639

47 Westwood, I., Cheary, D. M., Baxter, J. E., Richards, M. W., van Montfort, R. L., Fry, A. M. and Bayliss, R. (2009) Insights into the conformational variability and regulation of human Nek2 kinase. J Mol Biol. 386, 476-485

48 Whelligan, D. K., Solanki, S., Taylor, D., Thomson, D. W., Cheung, K. M., Boxall, K., Mas-Droux, C., Barillari, C., Burns, S., Grummitt, C. G., Collins, I., van Montfort, R. L., Aherne, G. W., Bayliss, R. and Hoelder, S. (2010) Aminopyrazine inhibitors binding to an unusual inactive conformation of the mitotic kinase Nek2: SAR and structural characterization. J Med Chem. 53, 7682-7698 49 Sidrauski, C. and Walter, P. (1997) The transmembrane kinase Ire1p is a site-specific endonuclease that initiates mRNA splicing in the unfolded protein response. Cell. 90, 1031-1039

50 Ali, M. M., Bagratuni, T., Davenport, E. L., Nowak, P. R., Silva-Santisteban, M. C., Hardcastle, A., McAndrews, C., Rowlands, M. G., Morgan, G. J., Aherne, W., Collins, I., Davies, F. E. and Pearl, L. H. (2011) Structure of the Ire1 autophosphorylation complex and implications for the unfolded protein response. EMBO J. 30, 894-905

51 Sanches, M., Duffy, N. M., Talukdar, M., Thevakumaran, N., Chiovitti, D., Canny, M. D., Lee, K., Kurinov, I., Uehling, D., Al-awar, R., Poda, G., Prakesch, M., Wilson, B., Tam, V., Schweitzer, C., Toro, A., Lucas, J. L., Vuga, D., Lehmann, L., Durocher, D., Zeng, Q., Patterson, J. B. and Sicheri, F. (2014) Structure and mechanism of action of the hydroxy-aryl-aldehyde class of IRE1 endoribonuclease inhibitors. Nat Commun. 5, 4202

52 Joshi, A., Newbatt, Y., McAndrew, P. C., Stubbs, M., Burke, R., Richards, M. W., Caldwell, J. J., McHardy, T., Collins, I. and Bayliss, R. (2015) Molecular mechanisms of human IRE1 activation through dimerization and ligand binding Oncotarget. (in press)

53 Lee, K. P., Dey, M., Neculai, D., Cao, C., Dever, T. E. and Sicheri, F. (2008) Structure of the dual enzyme Ire 1 reveals the basis for catalysis and regulation in nonconventional RNA splicing. Cell. 132, 89-100

54 Korennykh, A. V., Egea, P. F., Korostelev, A. A., Finer-Moore, J., Zhang, C., Shokat, K. M., Stroud, R. M. and Walter, P. (2009) The unfolded protein response signals through high-order assembly of Ire1. Nature. 457, 687-693 
55 Korennykh, A. and Walter, P. (2012) Structural basis of the unfolded protein response. Annu Rev Cell Dev Biol. 28, 251-277

56 Manning, G., Whyte, D. B., Martinez, R., Hunter, T. and Sudarsanam, S. (2002) The protein kinase complement of the human genome. Science. 298, 1912-1934

57 Villa, F., Deak, M., Alessi, D. R. and van Aalten, D. M. (2008) Structure of the OSR1 kinase, a hypertension drug target. Proteins. 73, 1082-1087

58 Padyana, A. K., Qiu, H., Roll-Mecak, A., Hinnebusch, A. G. and Burley, S. K. (2005) Structural basis for autoinhibition and mutational activation of eukaryotic initiation factor 2alpha protein kinase GCN2. J Biol Chem. 280, 29289-29299

59 Artim, S. C., Mendrola, J. M. and Lemmon, M. A. (2012) Assessing the range of kinase autoinhibition mechanisms in the insulin receptor family. Biochem J. 448, 213-220

60 Russo, A. A., Jeffrey, P. D. and Pavletich, N. P. (1996) Structural basis of cyclin-dependent kinase activation by phosphorylation. Nat Struct Biol. 3, 696700

61 Schulze-Gahmen, U., De Bondt, H. L. and Kim, S. H. (1996) High-resolution crystal structures of human cyclin-dependent kinase 2 with and without ATP: bound waters and natural ligand as guides for inhibitor design. J Med Chem. 39, 4540-4546 


\section{Figure legends}

Figure 1. Kinase activation as a multi-step process. In an autoinhibited state (grey) the kinase is catalytically inactive and unable to phosphorylate itself or other substrates. An external stimulus such as protein partner binding releases autoinhibition. In a pre-active state (blue), a kinase is able to phosphorylate itself, but is inefficient at phosphorylation of other substrates. Phosphorylation of the kinase results in a fully active state (orange) that acts on protein substrates.

Figure 2. Conformational changes in CDK2. A) Crystal structure of active CDK2, phosphorylated on Thr160 and bound to Cyclin-A (PDB ID: 1JST) [60]. B) Crystal structure of inactive CDK2 (PDB ID: 1HCK) [61]. C) Magnified view of (A) showing CDK2 active site. D) Magnified view of (B) showing CDK2 active site. In panels $A$ and $C$, active CDK2 is coloured orange, Cyclin-A is coloured yellow and phosphorylated T160 is coloured blue. In panels B and D, inactive CDK2 is coloured grey. In panels $C$ and $D$, the salt bridge residues are coloured red and the 3 basic residues that interact with T160 are coloured blue and labeled (i,ii,iii). In all panels the hydrophobic R-spine residues are coloured green and the ligand bound to the active site is coloured light beige.

Figure 3. Nek7 has a Tyr-down autoinhibitory mechanism. A) Crystal structure of inactive Nek7 showing the overall conformation, ADP (light beige) bound to the active site and the R-spine residues (green) (PDB ID: 2WQN) [33]. B) Magnified view of Nek7 showing the Lys-Glu residues (red), basic residues that are predicted to interact with phosphorylated Ser195 (blue) and the gatekeeper Leu111 (orange). C) Magnified view of the crystal structure of active TA02 (PDB ID: 1U5R) [35]. Key residues are colour-coded the same as their equivalents in Nek7 shown in panel (B).

Figure 4. Conformational plasticity of Nek2 in response to inhibitor binding. A) Crystal structure of Nek2 in a CDK2/SRC-like $\alpha$ C-helix out conformation bound to a pyrrole-indolino inhibitor (PDB ID: 2JAV) [45]. B) Crystal structure of Nek2 in a DFG-out conformation bound to a hybrid inhibitor (PDB ID: 4A4X) [44]. C) Crystal structure of Nek2 bound to ADP (PDB ID: 2W5A) [47]. D) Crystal structure of Nek2 in a Tyr-down conformation bound to an aminopyrazine inhibitor (PDB ID: 2XKF) [48]. The side chain -OH group of Tyr70 forms a H-bond with the main chain at the DFG-motif (black dashed line).

Figure 5. IRE1 has a Tyr-down autoinhibitory mechanism. A) Crystal structure of unphosphorylated ADP-bound human IRE1 in a Tyr-down, autoinhibited conformation (PDB ID: 3P23) [50] showing the R-spine residues (green), Lys-Glu residues (red) and basic residues that are predicted to interact with phosphorylated activation loop (blue). B) Crystal structure of unphosphorylated apo-form human IRE1 in a Tyr-up, pre-active conformation (PDB ID: 4Z7G) [52] C) Crystal structure of phosphorylated yeast IRE1 in a fully active conformation (PDB ID: 3FBV) [54]. 
Figure 6. The potential for Tyr-down autoinhibition is unevenly

distribution across the human kinome. A) Classification of human kinases that have a tyrosine as the residue on the $\beta 4$-strand within the R-spine. Kinases are classified into major groups as defined by Manning and colleagues [56]. Red circles indicate major groups or families that include kinases with the potential for Tyr-down autoinhibition. The size of the red circles is determined by the indicated number of relevant kinases. The "other" group has been split into families. B) Crystal structure of OSR1 kinase showing Tyr-down conformation (PDB ID: 2VWI, chain A) and C) Tyr-up conformation (PDB ID: 2VWI, chain B). D) Crystal structure of GCN2 kinase showing Tyr-down conformation (PDB ID: 1ZYC, chain A). 

Table 1. Classification of protein kinases with the potential for regulation by a Tyr-down autoinhibitory mechanism

\begin{tabular}{|c|c|c|}
\hline Name & Family & Group \\
\hline VRK1 & VRK & CK1 \\
\hline IRE1 & IRE & Other \\
\hline IRE2 & IRE & Other \\
\hline AAK1 & NAK & Other \\
\hline BIKE/BMP2 & NAK & Other \\
\hline NEK1 & NEK & Other \\
\hline NEK10 & NEK & Other \\
\hline NEK2 & NEK & Other \\
\hline NEK4 & NEK & Other \\
\hline NEK6 & NEK & Other \\
\hline NEK7 & NEK & Other \\
\hline NEK8 & NEK & Other \\
\hline NEK9 & NEK & Other \\
\hline SgK071/STKLD1 & Other-Unique & Other \\
\hline GCN2/EIF2AK4 & PEK & Other \\
\hline PEK & PEK & Other \\
\hline PKR & PEK & Other \\
\hline SCYL1 & SCY1 & Other \\
\hline TBCK & TBCK & Other \\
\hline PBK & TOPK & Other \\
\hline WEE1 & WEE & Other \\
\hline WEE2/WEE1B & WEE & Other \\
\hline CYGD/GUCY2D & RGC & RGC \\
\hline GCN2/EIF2AK4 & STE-Unique & STE \\
\hline ASK1/MAP3K5 & STE11 & STE \\
\hline МАР3К15/MAР3К7 & STE11 & STE \\
\hline МАР3К19/МАР3К8 & STE11 & STE \\
\hline MAP3K2 & STE11 & STE \\
\hline MAP3K3 & STE11 & STE \\
\hline MAP3K4 & STE11 & STE \\
\hline MAP3K6 & STE11 & STE \\
\hline GCK/MAP4K2 & STE20 & STE \\
\hline KHS1/MAP4K5 & STE20 & STE \\
\hline KHS2/MAP4K3 & STE20 & STE \\
\hline MAP4K1/HPK1 & STE20 & STE \\
\hline MST1 & STE20 & STE \\
\hline MST2 & STE20 & STE \\
\hline MST3 & STE20 & STE \\
\hline MST4 & STE20 & STE \\
\hline OSR1 & STE20 & STE \\
\hline PAK1 & STE20 & STE \\
\hline PAK3 & STE20 & STE \\
\hline STK25/YSK1 & STE20 & STE \\
\hline STK39/STLK3 & STE20 & STE \\
\hline STLK5/STRADA & STE20 & STE \\
\hline STLK6/STRADB & STE20 & STE \\
\hline TAOK1 & STE20 & STE \\
\hline TAOK2 & STE20 & STE \\
\hline TAOK3 & STE20 & STE \\
\hline ZC1/HGK/MAP4K4 & STE20 & STE \\
\hline ZC2/TNIK & STE20 & STE \\
\hline ZC3/MINK & STE20 & STE \\
\hline JAK1 & JakA & TK \\
\hline
\end{tabular}




\begin{tabular}{|l|l|l|}
\hline JAK2 & JakA & TK \\
\hline JAK3 & JakA & TK \\
\hline TYK2 & JakA & TK \\
\hline
\end{tabular}




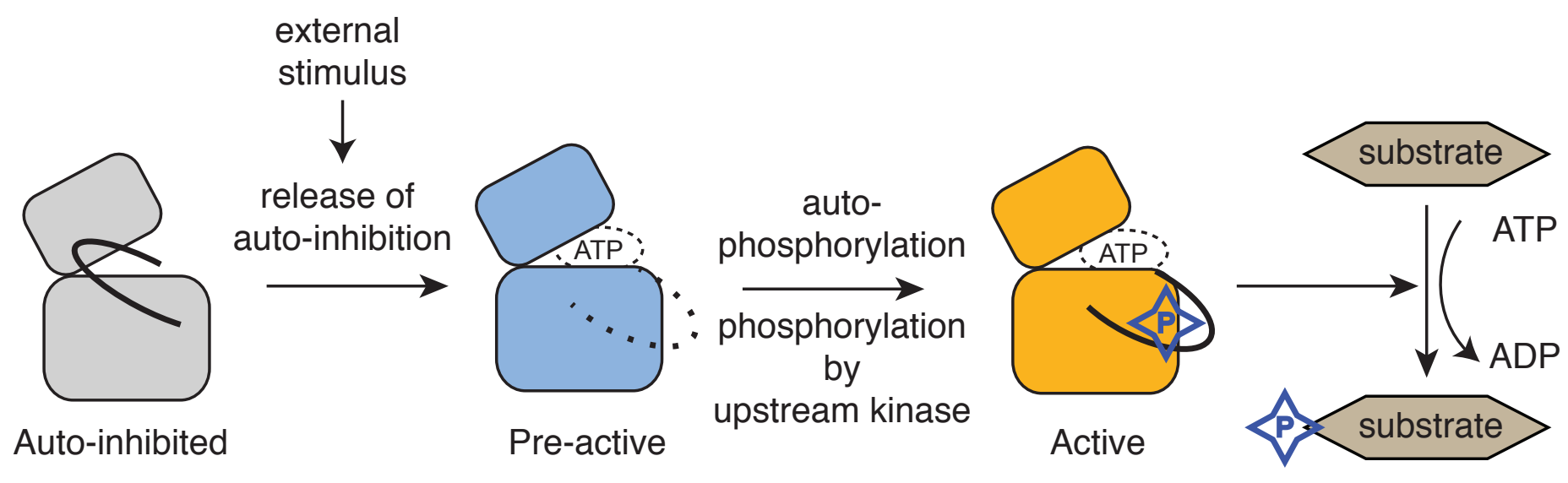




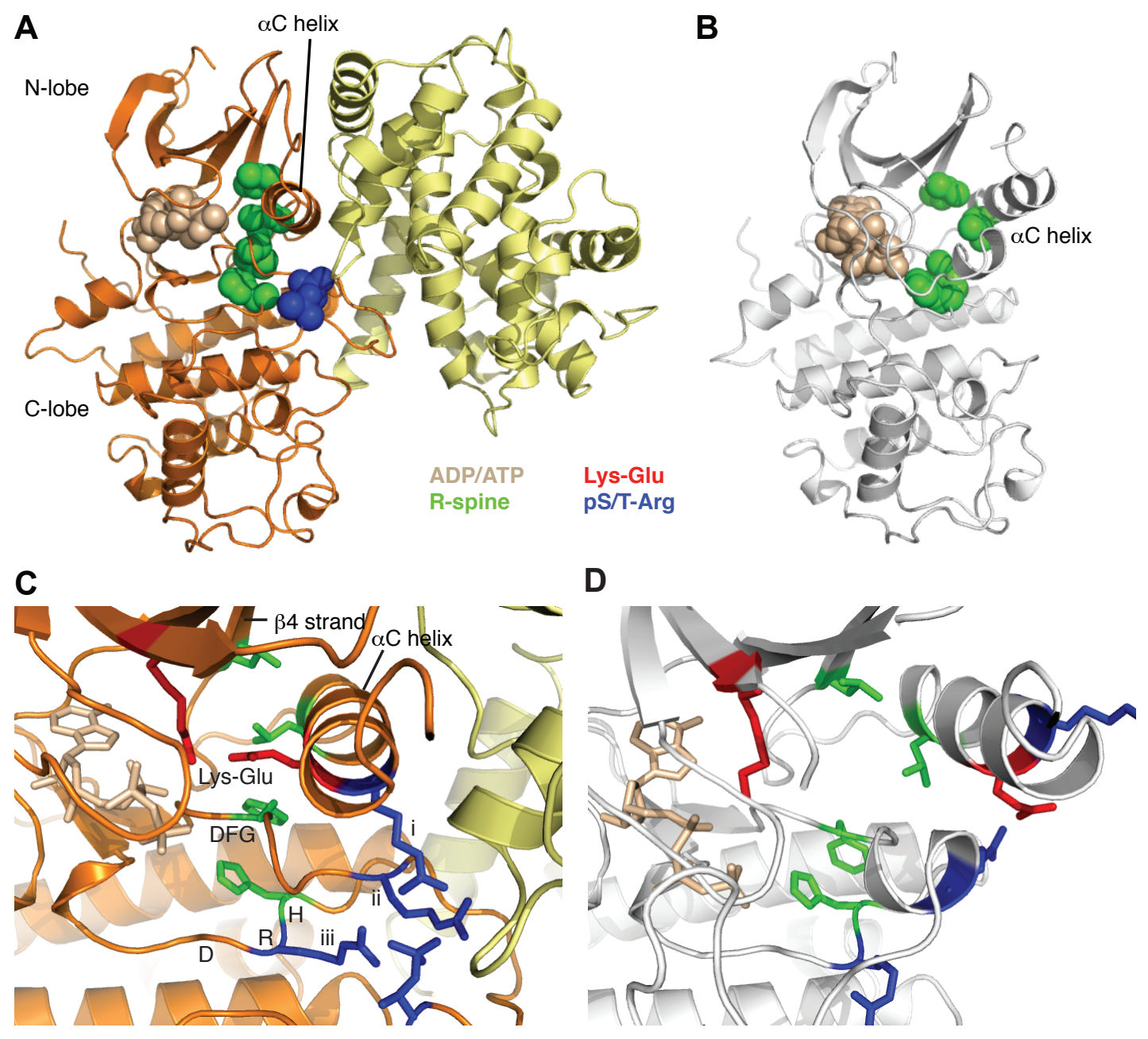



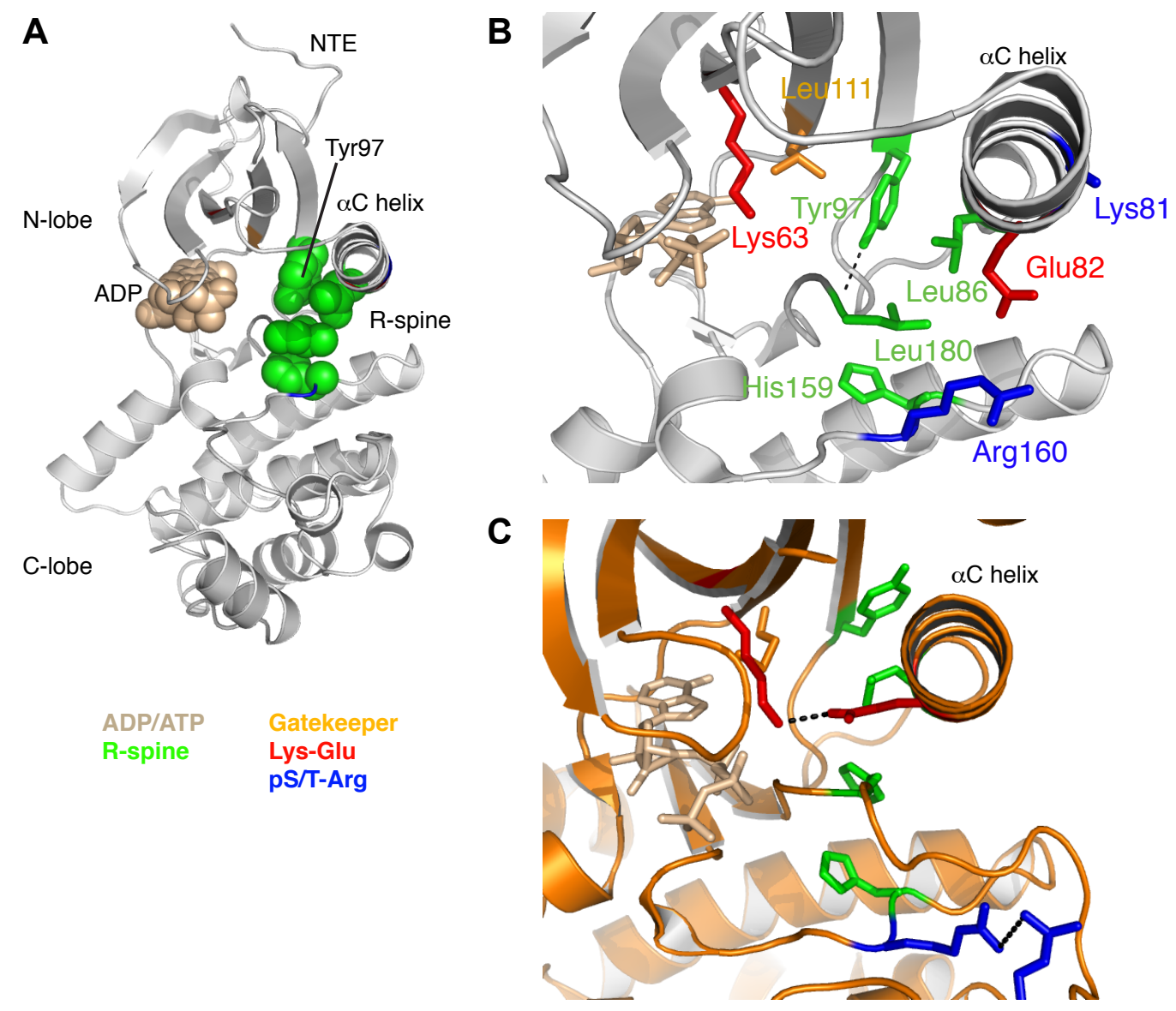

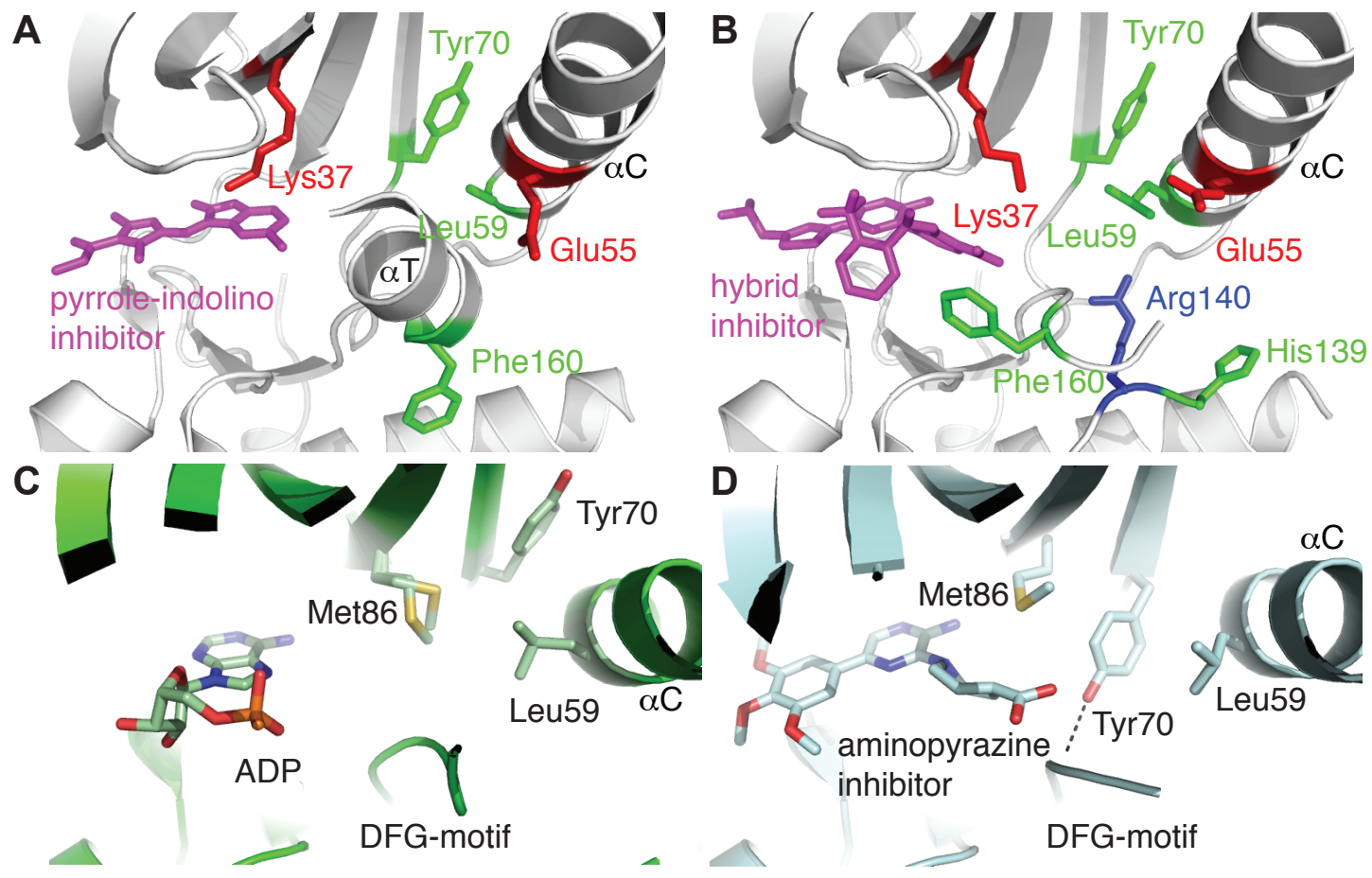

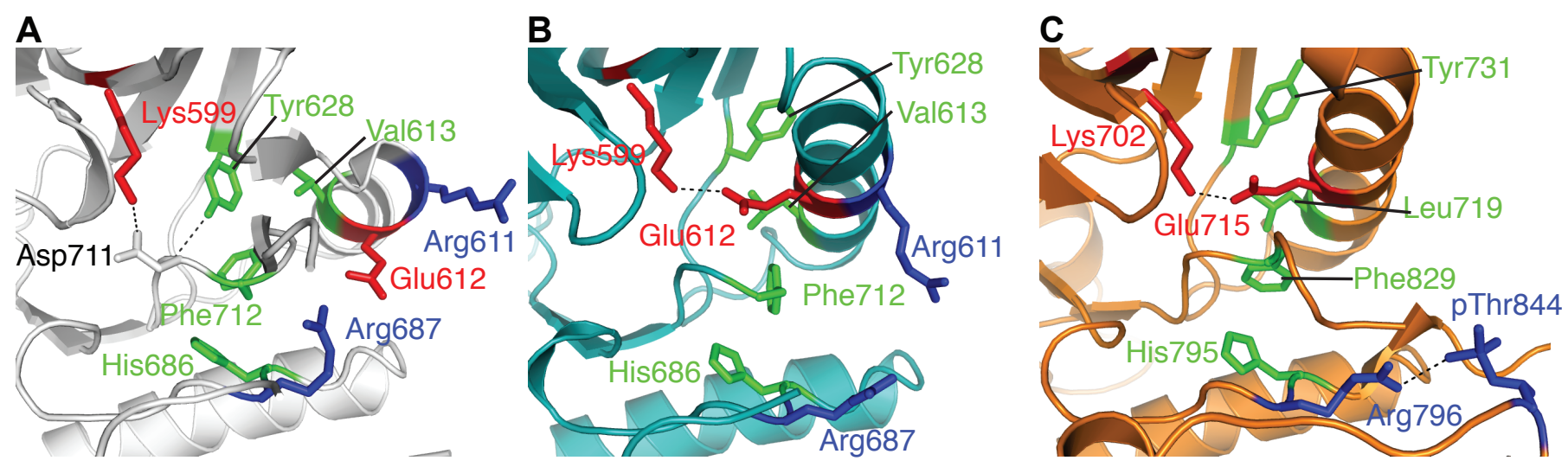

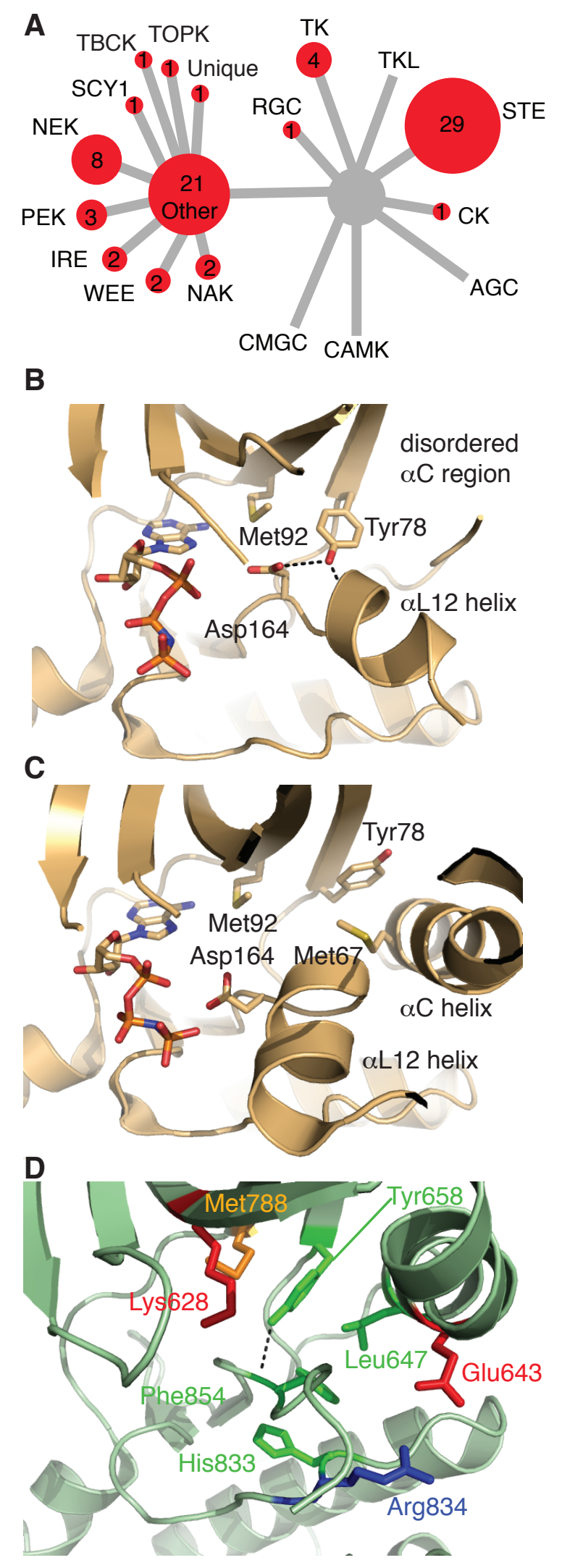